\title{
Enzalutamide in chemotherapy-naive patients with metastatic castration-resistant prostate cancer: A retrospective Korean multicenter study in a real-world setting
}

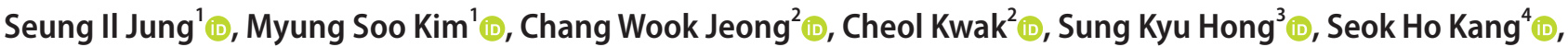 \\ Jae Young Joung ${ }^{5}(1)$, Seung Hwan Lee ${ }^{6}$, Seok Joong Yun ${ }^{7}$, Tae-Hwan Kim ${ }^{8}$ (i), Sung Woo Park ${ }^{9}$,

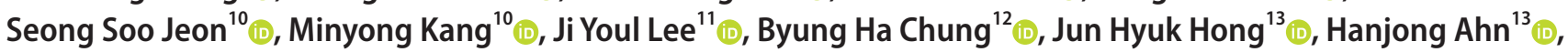 \\ Choung-Soo Kim ${ }^{13}$, Dong Deuk Kwon ${ }^{1}$ (i) \\ ${ }^{1}$ Department of Urology, Chonnam National University Medical School, Gwangju, ${ }^{2}$ Department of Urology, Seoul National University Hospital, Seoul National University \\ College of Medicine, Seoul, ${ }^{3}$ Department of Urology, Seoul National University Bundang Hospital, Seoul National University College of Medicine, Seongnam, ${ }^{4}$ Department \\ of Urology, Korea University College of Medicine, Seoul, ${ }^{5}$ Department of Urology, Center for Prostate Cancer, Korea National Cancer Center, Goyang, Department of \\ Urology, Severance Hospital, Yonsei University Health System, Seoul, 'Department of Urology, Chungbuk National University Hospital, Chungbuk National University \\ College of Medicine, Cheongju, ${ }^{8}$ Department of Urology, Kyungpook National University School of Medicine, Daegu, ${ }^{9}$ Department of Urology, Pusan National University \\ Yangsan Hospital, Yangsan, ${ }^{10}$ Department of Urology, Samsung Medical Center, Sungkyunkwan University School of Medicine, Seoul, ${ }^{11}$ Department of Urology, The \\ Catholic University of Korea, Seoul St. Mary Hospital's, College of Medicine, The Catholic University of Korea, Seoul, ${ }^{22}$ Department of Urology, Gangnam Severance \\ Hospital, Yonsei University College of Medicine, Seoul, ${ }^{13}$ Department of Urology, Asan Medical Center, University of Ulsan College of Medicine, Seoul, Korea
}

Purpose: This study aimed to evaluate the clinical efficacy of enzalutamide in chemotherapy-naïve metastatic castration-resistant prostate cancer (mCRPC) patients using real-world data from Korean patients.

Materials and Methods: We retrospectively reviewed the medical records of 199 chemotherapy-naïve patients with mCRPC at 13 tertiary centers in Korea between 2014 and 2017. All patients received enzalutamide daily and 89 patients received concurrent androgen deprivation therapy (ADT).

Results: The median age of the patients was 74 years. Initial results showed that $81.5 \%$ of the patients had Gleason score $\geq 8$ and $33.3 \%$ of the patients had European Cooperative Oncology Group Performance Status 0 . The overall mortality rate was $12 \%$. The median OS was not archieved and $76.7 \%$ of patients were alive at 30 months. Median time until PSA progression was 6 months. The overall survival rate at 2 years was significantly higher $(84.6 \%$ vs. $71.7 \%, p=0.015)$ and the duration of PSA progression-free survival was significantly longer ( 8.0 vs. 4.6 months, $p=0.008$ ) in patients receiving concurrent ADT than in those receiving enzalutamide alone. The incidence of adverse events of grade 3 or higher was 1.7\%. Multivariate Cox proportional hazard analysis indicated that ADT administered concurrently with enzalutamide significantly improved the overall survival (hazard ratio, $0.346 ; 95 \%$ confidence interval, 0.125-0.958).

Conclusions: Enzalutamide is effective and safe for chemotherapy-naïve patients with mCRPC. Furthermore, the overall survival was significantly higher in patients receiving enzalutamide and concurrent ADT than in patients receiving enzalutamide alone.

Keywords: Androgen antagonists; Neoplasm metastasis; Prostate neoplasms; Treatment outcome

This is an Open Access article distributed under the terms of the Creative Commons Attribution Non-Commercial License (http://creativecommons.org/licenses/by-nc/4.0) which permits unrestricted non-commercial use, distribution, and reproduction in any medium, provided the original work is properly cited.

Received: 10 December, 2018 - Accepted: 25 August, 2019

Corresponding Author: Dong Deuk Kwon (iD https://orcid.org/0000-0002-4544-5420

Department of Urology, Chonnam National University Hospital, Chonnam National University Medical School, 42 Jebong-ro, Dong-gu, Gwangju 61469, Korea

TEL: +82-62-220-6700, FAX: +82-62-227-1643, E-mail: urokwon@gmail.com 


\section{INTRODUCTION}

In 2012, prostate cancer was the fifth most commonly diagnosed malignancy in Korean men [1]. The incidence of prostate cancer is lower in Korea than in western countries [2]. However, a steady increase has been observed in the prevalence of prostate cancer in Korea during the last decade [3]. Progression of prostate cancer leads to metastasis, and most patients respond to androgen deprivation therapy (ADT). However, patients develop resistance to ADT, and this condition is known as metastatic castration-resistant prostate cancer (mCRPC). Metastatic CRPC is defined by progression of cancer despite the effective lowering of serum testosterone levels to castrate levels [4]. Docetaxel plus prednisone is the current standard of care for MCRPC in Korea, but this therapy has toxic effects and leads to the development of drug resistance [5]. Recent findings have shown that androgen receptor signaling plays an important role in CRPC, which suggests that inhibition of this pathway using non-cytotoxic therapies may confer a survival benefit. The results of the international, randomized, double-blind, phase 3, PREVAIL trial in chemotherapy-naïve men showed that enzalutamide improves overall survival (OS) [6].

Results of the PREVAIL showed that treatment with enzalutamide showed positive outcomes on all secondary endpoints, including the time until the first skeletal-related event (SRE), soft-tissue response rate, time until prostatespecific antigen (PSA) progression, and PSA response rate ( $\geq 50 \%$ decline), as well as patient-reported outcomes [6-9]. Enzalutamide was approved in Korea in June 2013 for use in men with CRPC post-chemotherapy based on the results of the AFFIRM trial. In May 2015, the indication was expanded to include men with CRPC regardless of previous exposure to chemotherapy based on results of the PREVAIL trial [10]. This study aimed to evaluate the clinical results of enzalutamide in chemotherapy-naïve mCRPC patients using Korean, multicenter, real-world data and to compare them with the results of the PREVAIL study. In Korea, continuous ADT for mCRPC patients was prohibited until January 2017; subsequently, concurrent ADT was allowed. Thus, we were able to analyze the efficacy of enzalutamide and concurrent $\mathrm{ADT}$ in the treatment of patients with chemotherapy-naïve mCRPC. This study will support evidence to the current recommendations for continuing luteinizing hormone-releasing hormone (LHRH) therapy, which can improve the benefits of enzalutamide treatment in patients with chemotherapy-naïve mCRPC.

\section{MATERIALS AND METHODS}

\section{Study design and patients}

We retrospectively reviewed the medical records of 199 chemotherapy-naïve patients with $\mathrm{mCRPC}$ at 13 tertiary centers in Korea between 2014 and 2017. The exclusion criteria were previous docetaxel chemotherapy, brain metastasis, or epidural disease. Patients who had already received enzalutamide treatment included in a clinical trial were excluded. Enzalutamide was administered daily at an initial dose of $160 \mathrm{mg}$, and ADT was administered in a conventional manner. Unlike the PREVAIL trial, our trial did not have stringent eligibility criteria to reflect a real clinical environment. The endpoint of this trial was OS, which was defined as the time from the start of treatment to any cause of death. We followed the protocol mentioned in the PREVAIL trial for the definition of response criteria and endpoints in Prostate Cancer Clinical Trial Working Group 2, Solid Tumor Version 1.1 (RECIST 1.1). For patients with a decreased PSA levels at week 13, the time to PSA progression was defined as the day on which PSA levels increased by more than $25 \%$ and the day on which PSA levels increased by at least $2 \mathrm{ng} / \mathrm{mL}$ over a minimum of two consecutive days. For patients without a decreased PSA levels at week 13, the day on which PSA levels increased by more than $25 \%$ and the day on which a $2 \mathrm{ng} / \mathrm{mL}$ increase over baseline levels was recorded was identified as the second consecutive value. Radiographic progression-free survival (rPFS) was defined as the time of initial objective evidence of radiological progression or death. Radiographic progression is defined as the appearance of two or more new lesions according to RECIST 1.1 or on the bone scan for soft tissue disease. SREs are defined as the events that necessitate changes in chemotherapy, such as radiation therapy or bone surgery for the treatment of pathological fractures, spinal cord compression, or bone pain. Advent events (AEs) were evaluated according to National Cancer Institute Common Terminology Criteria for Adverse Events, version 4.03.

\section{Data collection and statistical analysis}

The research data were collected using the Research Electronic Data Capture (REDCap) program designed to support data capture. We performed the chi-square or MannWhitney U tests to compare the categorical and continuous variables, respectively, between the two groups. PFS and OS were analyzed using Kaplan-Meier methods and log-rank tests. Univariate and multivariate Cox proportional hazards regression analyses were performed to assess the association between baseline parameters and OS. All p-values correspond 
to two-sided tests, with $p$-values $<0.05$ considered statistically significant. PSA response summaries were presented as percentages of patients who showed a decrease in PSA levels by more than $50 \%$ or $90 \%$, respectively, from baseline to lowest point, and were visualized using waterfall plots. AEs were described as the rate of all grade or grade $\geq 3$ events.

\section{Ethical statement}

This study was approved by the Institutional Review Boards at each participating center (representative IRB: Seoul National University Hospital IRB no. H-1604-099-755). Subjects provided written informed consent, and the study was performed according to the Declaration of Helsinki.

\section{RESULTS}

Demographic features and disease characteristics of patients are shown in Table 1 [6,10]. Initial results showed that $81.5 \%$ of the patients had Gleason score (GS) $\geq 8$ and $33.3 \%$ of the patients had European Cooperative Oncology Group Performance Status (ECOG PS) 0. Eighty-nine patients (44.9\%) received concurrent ADT. Median follow-up is 9.4 months (interquartile range, 3.9-13.5 months). The overall mortality rate was $12 \%$. The Kaplan-Meier curves for OS and rPFS are shown in Fig. 1 [6,10]. Median OS, median rPFS, median time until chemotherapy, median time until first SRE was not reached until our analysis (Fig. 1, Table 2) [6,10]. Median time until PSA progression was 6 months (Table 2) [6,10]. After enzalutamide treatment, $35.2 \%$ of patients received more than one systemic chemotherapy (Table 3) [6,10]. The median OS was not achieved and $76.7 \%$ of patients were alive at 30 months. In a Kaplan-Meier curve with $95 \%$ confidence interval (CI), OS was 0.84 (95\% CI, 0.76-0.91) at 12 months and 0.77 (95\% CI, 0.67-0.87) at 24 months (Supplementary Fig. 1). The results of the univariate Cox proportional hazard analysis for OS showed that concurrent ADT, ECOG PS, and baseline PSA levels were significant predictors of OS (Table 4). Results of multivariate analysis showed that enzalutamide and concurrent ADT significantly improved the OS (hazard ratio, 0.346; 95\% CI, 0.125-0.958). The median duration of enzalutamide treatment was 5 months, and the incidence of AEs of grade 3 or higher was 1.7\% (Table 5) [6,10]. The Kaplan-Meier curves for OS, PSA PFS, and rPFS after concurrent ADT are shown in Fig. 2. The OS by 2 years was significantly improved with concurrent ADT (84.6\% vs. $71.7 \%, \mathrm{p}=0.015)$. In addition, patients receiving concurrent ADT showed a significant improvement in PSA PFS (8.0 vs. 4.6 months, $p=0.008$ ). However, rPFS at 2 years was not dif-

Table 1. Baseline patient and disease characteristics

\begin{tabular}{|c|c|c|c|c|c|}
\hline \multirow{2}{*}{ Characteristic } & \multicolumn{2}{|c|}{ PREVAIL $(n=1,717)[6]$} & \multicolumn{2}{|c|}{ PREVAIL Korean $(n=78)[10]$} & \multirow{2}{*}{$\begin{array}{l}\text { Current study } \\
\qquad(n=199)\end{array}$} \\
\hline & ENZA $(n=872)$ & Placebo $(n=845)$ & ENZA $(n=40)$ & Placebo $(n=38)$ & \\
\hline Median age (y) & $72(43-93)$ & $71(42-93)$ & $71(56-83)$ & $67(42-85)$ & $74(51-94)$ \\
\hline Median body weight (kg) & 83.1 & 82.8 & 67.0 & 70.5 & 66.0 \\
\hline Body mass index $\left(\mathrm{kg} / \mathrm{m}^{2}\right)$ & 27.5 & 27.5 & 24.4 & 25.5 & 24.2 \\
\hline Gleason score $\geq 8$ at initial diagnosis & $424(50.6)$ & $423(52.4)$ & $30(81.1)$ & $31(81.6)$ & $145(81.5)$ \\
\hline Missing & 34 & 37 & 3 & & 21 \\
\hline ECOG $P S$ grade $=0$ & $584(67.0)$ & $585(69.2)$ & $22(55.0)$ & $29(76.3)$ & $62(33.3)$ \\
\hline Missing & & & & & 13 \\
\hline Median PSA (ng/mL) & 54.1 & 44.2 & 25.4 & 26.2 & 51.6 \\
\hline Median LDH (IU/L) & 185.0 & 185.0 & 191.0 & 184.5 & 333.0 \\
\hline Prior radical prostatectomy (\%) & $226(25.9)$ & $225(26.6)$ & $11(27.5)$ & $4(10.5)$ & $36(18.2)$ \\
\hline Missing & & & & & 1 \\
\hline Bone disease (\%) & $741(85.0)$ & $690(81.7)$ & $36(90.0)$ & $30(78.9)$ & $160(82.5)$ \\
\hline Missing & & & & & 5 \\
\hline$\geq 20$ bone metastases & $145(16.6)$ & $150(17.8)$ & Unknown & Unknown & $15(9.9)$ \\
\hline Missing & & & & & 47 \\
\hline Soft-tissue disease (lymph node, visceral, or other) & $517(59.3)$ & $504(59.6)$ & $22(55.0)$ & $22(57.9)$ & $110(58.5)$ \\
\hline Missing & & & & & 11 \\
\hline Concurrent ADT & $872(100.0)$ & $845(100.0)$ & $40(100.0)$ & $38(100.0)$ & $89(44.9)$ \\
\hline Missing & & & & & 1 \\
\hline
\end{tabular}

Values are presented as median (range), number only, or number (\%).

ENZA, enzalutamide; ECOG PS, European Cooperative Oncology Group Performance Status; PSA, prostate-specific antigen; LDH, lactate dehydrogenase; ADT, androgen deprivation therapy. 
A

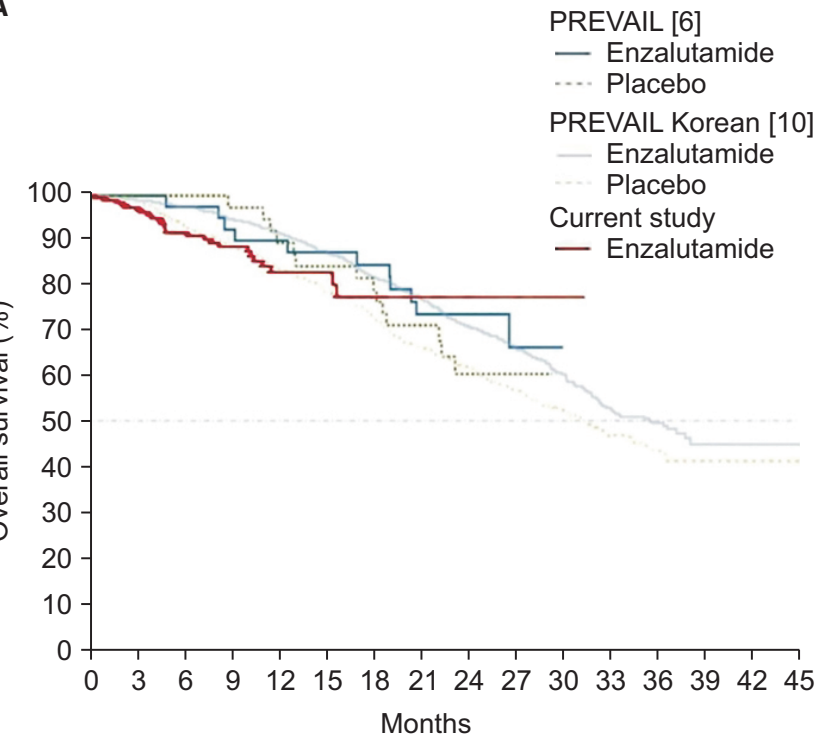

B

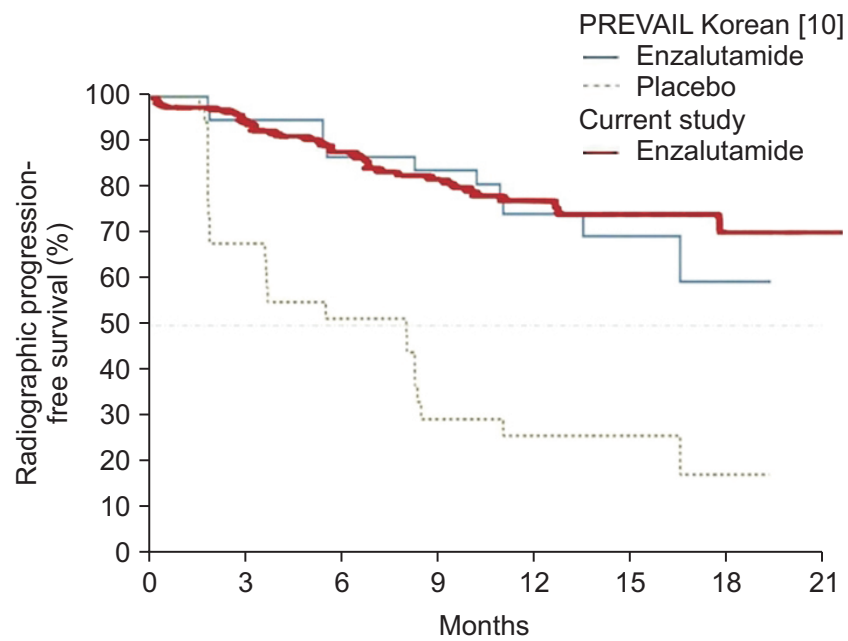

Fig. 1. Kaplan-Meier survival curves for (A) overall survival and (B) radiographic progression-free survival in PREVAIL study [6], in PREVAIL Korean post hoc analysis [10], and in this study.

Table 2. Summary of oncologic outcomes

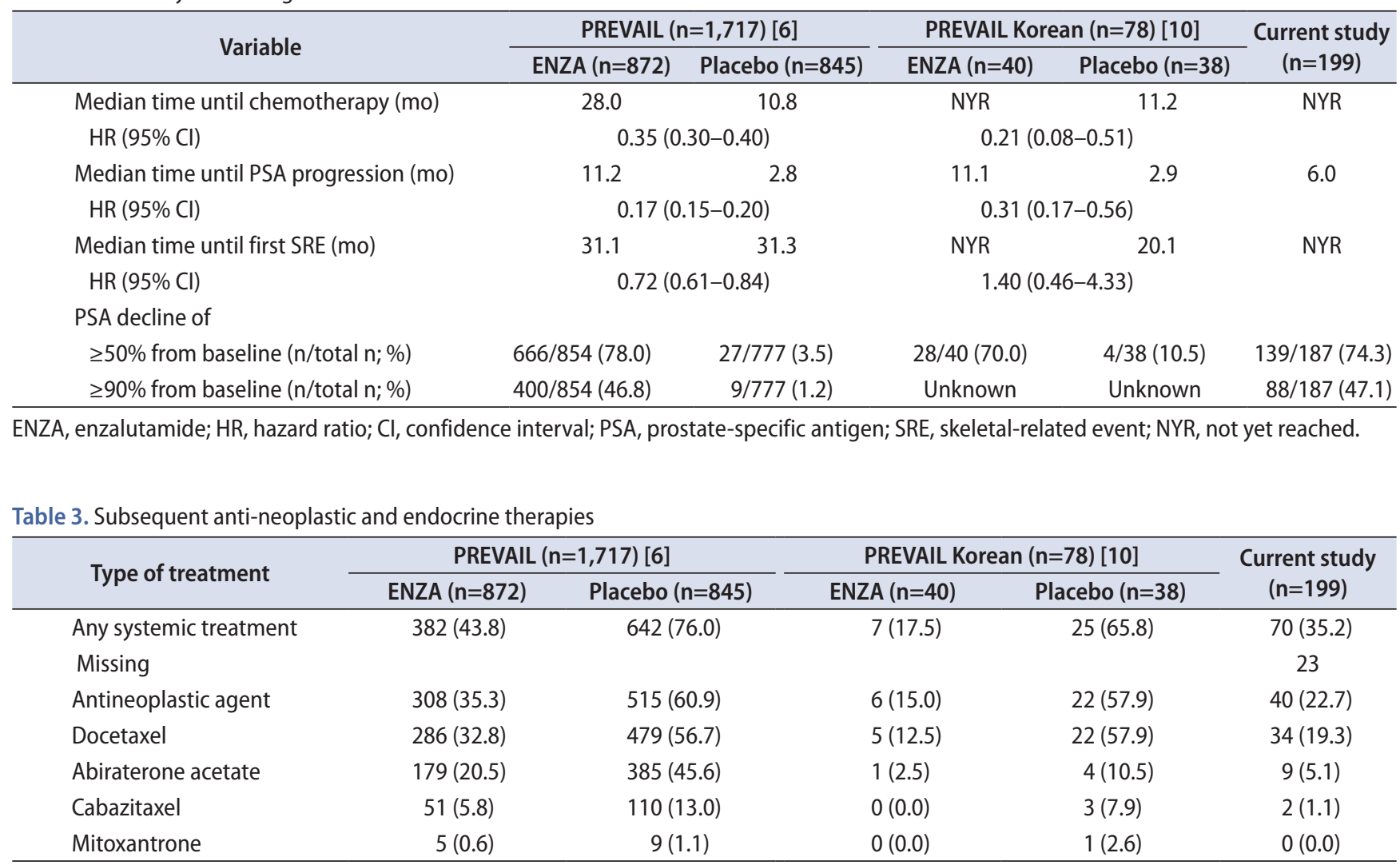

Duplicated count was allowed.

ENZA, enzalutamide.

ferent in patients receiving concurrent $\mathrm{ADT}$ (71.8\% vs. $68.4 \%$, $\mathrm{p}=0.154$ ). The waterfall plot of the change in PSA levels in patients receiving concurrent ADT is shown in Fig. 3. To compare Korean real-world data with the result of the PRE-
VAIL study, we described data of PREVAL study in Table 1, Table 2, Table 3, Table 5 and Fig. 1 [6,10]. 
Table 4. Cox regression analysis for overall survival

\begin{tabular}{|c|c|c|c|c|c|c|}
\hline \multirow{2}{*}{ Variable } & \multicolumn{3}{|c|}{ Univariate } & \multicolumn{3}{|c|}{ Multivariate } \\
\hline & HR & $95 \% \mathrm{Cl}$ & p-value & HR & $95 \% \mathrm{Cl}$ & p-value \\
\hline \multicolumn{7}{|l|}{ Age (y) } \\
\hline$<65$ & Reference & & & & & \\
\hline$\geq 65$ & 0.808 & $0.302-2.162$ & 0.672 & & & \\
\hline \multicolumn{7}{|l|}{ ECOG PS } \\
\hline $0-1$ & Reference & & & & & \\
\hline$\geq 2$ & 2.993 & $1.160-7.723$ & 0.023 & & & \\
\hline \multicolumn{7}{|l|}{ No. of HT } \\
\hline$\leq 2$ & Reference & & & & & \\
\hline$>2$ & 1.048 & $0.247-4.453$ & 0.949 & & & \\
\hline \multicolumn{7}{|l|}{ Type of progression at study entry } \\
\hline PSA only & Reference & & & & & \\
\hline Radiographic progression +/- PSA progression & 0.699 & $0.350-2.020$ & 0.699 & & & \\
\hline \multicolumn{7}{|l|}{ No. of bone lesions } \\
\hline$<20$ & Reference & & & & & \\
\hline$\geq 20$ & 1.090 & $0.141-8.435$ & 0.934 & & & \\
\hline \multicolumn{7}{|l|}{ Lymph node involvement } \\
\hline No & Reference & & & & & \\
\hline Yes & 0.943 & $0.415-2.139$ & 0.888 & & & \\
\hline \multicolumn{7}{|l|}{ Visceral metastasis } \\
\hline No & Reference & & & & & \\
\hline Yes & 1.170 & $0.342-4.006$ & 0.802 & & & \\
\hline \multicolumn{7}{|l|}{ Gleason score } \\
\hline$\leq 7$ & Reference & & & & & \\
\hline$\geq 8$ & 6.059 & $0.818-44.86$ & 0.078 & & & \\
\hline \multicolumn{7}{|l|}{ Baseline PSA } \\
\hline$\leq$ Median & Reference & & & Reference & & \\
\hline >Median & 2.517 & $1.084-5.840$ & 0.032 & 2.507 & $0.958-6.563$ & 0.061 \\
\hline \multicolumn{7}{|l|}{ Concurrent ADT } \\
\hline No & Reference & & & Reference & & \\
\hline Yes & 0.335 & $0.133-0.845$ & 0.020 & 0.346 & $0.125-0.958$ & 0.041 \\
\hline
\end{tabular}

HR, hazard ratio; Cl, confidence interval; ECOG PS, European Cooperative Oncology Group Performance Status; HT, hormonal treatment; PSA, prostate-specific antigen; ADT, androgen deprivation therapy.

\section{DISCUSSION}

The effects of enzalutamide observed in our study with asymptomatic or mildly symptomatic Korean chemotherapynaïve patients with mCRPC were consistent with those observed in the study population of the PREVAL trial [6,10].

However, the baseline disease characteristics of the subjects in our study were different from those of the subjects in the PREVAIL trial, which may be because of the differences in real clinical practice. A high percentage of Korean patients had a GS of at least 8, which indicates a high disease burden. Our finding was consistent with that observed in the PREVAIL trial in that $50.6 \%$ and $81.1 \%$ patients receiving enzalutamide among the overall population and in Korean subgroup, respectively, has a GS $\geq 8$ [10]. Some dif- ferences were observed in the characteristics of the disease between the real-world Korean patients and the subjects in the PREVAIL trial; these differences may be related to differences in ethnicity. Compared to the overall study population of the PREVAIL trial, a larger proportion of Korean patients had poor PS. A lower proportion of patients in our study had ECOG PS=0 (33.3\% vs. $67.0 \%$ in the PREVAIL study). In clinical practice, the physicians, patients, and relatives of chemotherapy-naïve patients with $\mathrm{mCRPC}$ with poor PS are reluctant to undergo chemotherapy. Therefore, they prefer treatment with enzalutamide despite its high cost before commencing chemotherapy. Thus, this finding may explain why our data showed that a high proportion of Korean patients had poor performance status. Korean patients receiving enzalutamide had a short median time 
Table 5. Summary of adverse events

\begin{tabular}{|c|c|c|c|c|c|}
\hline \multirow{2}{*}{ Parameter } & \multicolumn{2}{|c|}{ PREVAIL $(n=1,715)[6]$} & \multicolumn{2}{|c|}{ PREVAIL Korean $(n=78)[10]$} & \multirow{2}{*}{$\begin{array}{c}\text { Current study } \\
(n=199)\end{array}$} \\
\hline & ENZA $(n=871)$ & Placebo $(n=844)$ & ENZA $(n=40)$ & Placebo $(n=38)$ & \\
\hline Median duration of treatment (mo) & 16.6 & 4.6 & 13.0 & 5.1 & 5.0 \\
\hline Any AEs & $844(96.9)$ & $787(93.2)$ & $37(92.5)$ & $30(78.9)$ & $59(34.9)$ \\
\hline Missing & & & & & 30 \\
\hline Serious AEs & $279(32.0)$ & $226(26.8)$ & $14(35.0)$ & $3(7.9)$ & $3(1.7)$ \\
\hline Missing & & & & & 22 \\
\hline Grade $\geq 3$ AEs & $374(42.9)$ & $313(37.1)$ & $13(32.5)$ & $4(10.5)$ & $3(1.8)$ \\
\hline Missing & & & & & 30 \\
\hline Drug-related grade $\geq 3 \mathrm{AEs}$ & $67(7.7)$ & $54(6.4)$ & $0(0.0)$ & $0(0.0)$ & $3(1.7)$ \\
\hline Missing & & & & & 23 \\
\hline \multicolumn{6}{|l|}{ Most common AEs } \\
\hline Musculoskeletal pain & $87(10.0)$ & $73(8.6)$ & $4(10.0)$ & $2(5.3)$ & $8(4.7)$ \\
\hline Fatigue & $310(35.6)$ & $218(25.8)$ & $8(20.0)$ & $4(10.5)$ & $6(3.6)$ \\
\hline Decreased appetite & $158(18.1)$ & $136(16.1)$ & Unknown & Unknown & $5(3.0)$ \\
\hline Constipation & $193(22.2)$ & $145(17.2)$ & $6(15.0)$ & $2(5.3)$ & $2(1.2)$ \\
\hline Diarrhea & $142(16.3)$ & $119(14.1)$ & Unknown & Unknown & $2(1.2)$ \\
\hline Hypertension & $117(13.4)$ & $35(4.1)$ & Unknown & Unknown & $2(1.2)$ \\
\hline Hot flush & $157(18.0)$ & $65(7.7)$ & $5(12.5)$ & $2(5.3)$ & $2(1.2)$ \\
\hline Headache & $91(10.4)$ & $59(7.0)$ & Unknown & Unknown & $2(1.2)$ \\
\hline Back pain & $235(27.0)$ & $187(22.2)$ & $7(17.5)$ & $3(7.9)$ & $1(0.6)$ \\
\hline Nausea & $201(23.1)$ & $190(22.5)$ & $6(15.0)$ & $4(10.5)$ & $1(0.6)$ \\
\hline Asthenia & $113(13.0)$ & $67(7.9)$ & Unknown & Unknown & $1(0.6)$ \\
\hline Upper respiratory infection & $53(6.1)$ & $30(3.6)$ & $8(20.0)$ & $3(7.9)$ & $0(0.0)$ \\
\hline
\end{tabular}

Values are presented as number only or number (\%).

ENZA, enzalutamide; $A E$, adverse event.

A

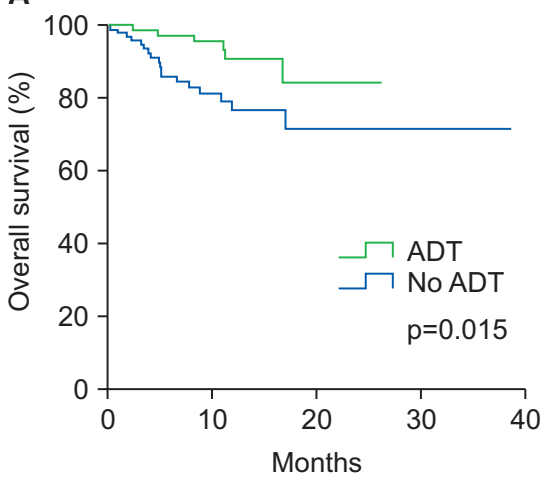

B

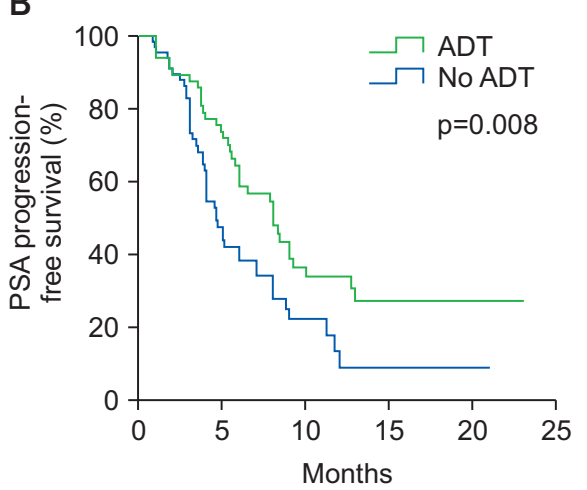

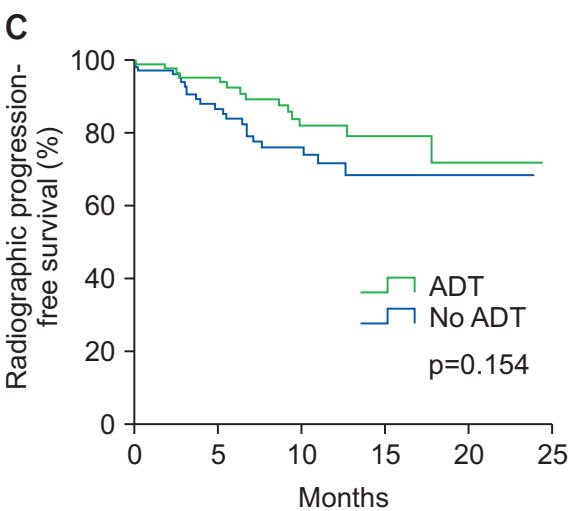

Fig. 2. According to concurrent androgen deprivation therapy (ADT), Kaplan-Meier survival estimates for (A) overall survival and (B) time to prostatespecific antigen (PSA) progression-free survival, and (C) radiographic progression-free survival.

until PSA progression, and poor baseline characteristics and PS may be attributed to this result. In addition to baseline characteristics, concurrent ADT affects the OS. Unlike the patients in the PREVAIL trial, a larger proportion of Korean patients (55\%) had did not receive concurrent ADT with enzalutamide in the clinical setting because the Korean medical insurance system prohibited continuous ADT for mCRPC until January 2017. Our results showed that ADT administered concurrently with enzalutamide improved the oncologic outcomes of chemotherapy-naïve patients with mCRPC.

To date, no study has established evidence supporting continuation of LHRH therapy for CRPC [11]. Few studies have investigated the effects of termination of LHRH therapy in patients with CRPC and, therefore, the current European guidelines recommend medical castration [12]. The Korean medical insurance system prohibited continuous ADT for mCRPC because of lack of evidence until January 2017. 


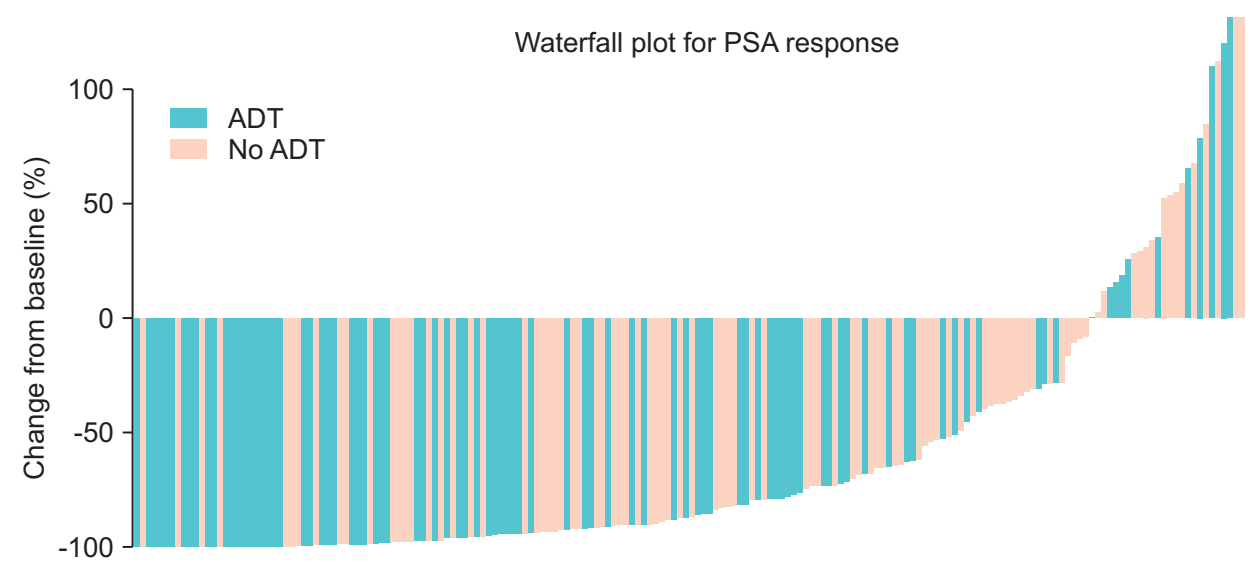

Fig. 3. Waterfall plot for prostate-specific antigen (PSA) response according to concurrent androgen deprivation therapy (ADT).
Continuation of LHRH therapy may cause considerable side effects and unnecessary expenses [13]. Results from previous clinical trials indicate that castration offers only a marginal survival benefit [14,15]. Taylor et al. [14] reported the results of a retrospective study, which showed a significant marginal survival benefit for patients continuing an LHRH agonist along with anthracycline, platinum, or ketoconazole treatment for mCRPC. However, a consensus remains to be achieved about the continuation of LHRH treatment. Analysis of 5 Southwest Oncology Group phase II chemotherapy trials conducted in the 1990s failed to show an obvious advantage of continuous ADT in terms of response to cytotoxic chemotherapy or OS in patients with CRPC [15]. The results of another retrospective study of 78 patients failed to show a survival benefit of docetaxel and concurrent ADT in patients with CRPC [16]. However, it could be an underpowered study because of the small number of patients. Lee et al. [16] found that serum testosterone levels did not recover to those observed in noncastrated individuals in most patients after withdrawal of ADT for the entire duration of chemotherapy. A similar phenomenon was observed in men with hormone-sensitive prostate cancer, in which approximately half of the patients who received ADT for a longterm remained castrated at 2.5 years after ADT cessation [17]. Results of previous studies indicate that serum testosterone levels remain low after long-term treatment with LHRH agonists [18-20]. Therefore, cessation of LHRH therapy may have only little clinical relevance since serum testosterone levels remain low and may not affect proliferation of cancer cells. However, unlike serum testosterone levels, serum LH levels recover after several weeks to months after cessation of LHRH therapy [18-20]. Cessation of LHRH therapy leads to rapid recovery of $\mathrm{LH}$ levels after a median of 58 days to 4.5 months, even after long-term medical castration $[18,19]$. Apart from its action on the testes and adrenal glands, $\mathrm{LH}$ acts directly on prostate cancer cells via $\mathrm{LH}$-specific receptors and increases the expression of several key steroidogenic enzymes [21]. Administration of abiraterone alone increases the LH [22]. To date, the SPARE trial (NCT02077634) is the only trial that provides further insights into the complex hormonal changes that occur after treatment with abiraterone with or without concurrent LHRH therapy [23]. The SPARE trial is investigating the role of continuation of LHRH therapy when starting treatment with abiraterone in patients with asymptomatic or mild symptomatic chemotherapynaïve CPRC. To date, no study has investigated the efficacy of enzalutamide and concurrent ADT in the treatment of chemotherapy-naïve mCPRC. Therefore, the results of our trial provide important evidence based on unique data.

This study is subject to the usual limitations inherent with retrospective design and data collection. The followup period of this study was relatively short. Further, some missing values are another weakness of the data. The rate of AEs in our study was lower than those in the PREVAIL trial, which may be because of the retrospective study design. The advantages of our study are that we analyzed realworld data from multiple institutions. Furthermore, the primary endpoint was OS, which is relatively objective and less confounded even in a retrospective study. Thus, our results provide novel data about the efficacy of enzalutamide and concurrent ADT for the treatment of men with chemotherapy-naïve mCRPC due to the previous medical environment in Korea, in which continuous ADT therapy for mCRPC patients was prohibited.

\section{CONCLUSIONS}

A higher proportion of patients in our cohort study showed a GS $\geq 8$ and poor ECOG performance status than those in the PREVAIL study, but the efficacy results in our study were similar to those in the PREVAIL study. Despite the limitations of retrospective studies, the safety 
was also acceptable. Enzalutamide is effective and safe for chemotherapy-naïve patients with mCRPC in the real-world setting. Further, compared to enzalutamide alone, concurrent administration of ADT with enzalutamide significantly prolonged the survival. Thus, we recommend concurrent administration of ADT with enzalutamide for the treatment of chemotherapy-naïve patients with mCRPC.

\section{CONFLICTS OF INTEREST}

The authors have nothing to disclose.

\section{AUTHORS' CONTRIBUTIONS}

Research conception and design: Chang Wook Jeong, Seung Il Jung, Cheol Kwak, and Dong Deuk Kwon. Data acquisition: all authors. Data analysis and interpretation: Myung Soo Kim, Chang Wook Jeong, and Seung Il Jung. Drafting of the manuscript: Myung Soo Kim and Seung Il Jung. Approval of final manuscript: all authors.

\section{SUPPLEMENTARY MATERIAL}

Scan this QR code to see the supplementary material, or visit https://www.icurology.org/src/sm/icurology-61-19-s001.pdf.

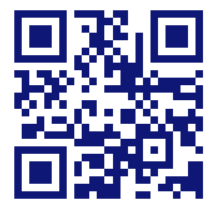

\section{REFERENCES}

1. Jung KW, Won YJ, Kong HJ, Oh CM, Cho H, Lee DH, et al. Cancer statistics in Korea: incidence, mortality, survival, and prevalence in 2012. Cancer Res Treat 2015;47:127-41.

2. Ferlay J, Soerjomataram I, Dikshit R, Eser S, Mathers C, Rebelo $\mathrm{M}$, et al. Cancer incidence and mortality worldwide: sources, methods and major patterns in GLOBOCAN 2012. Int J Cancer 2015;136:E359-86.

3. Lee DH, Jung HB, Chung MS, Lee SH, Chung BH. The change of prostate cancer treatment in Korea: 5 year analysis of a single institution. Yonsei Med J 2013;54:87-91.

4. Scher HI, Halabi S, Tannock I, Morris M, Sternberg CN, Carducci MA, et al.; Prostate Cancer Clinical Trials Working Group. Design and end points of clinical trials for patients with progressive prostate cancer and castrate levels of testosterone: recommendations of the Prostate Cancer Clinical Trials Working Group. J Clin Oncol 2008;26:1148-59.
5. Tannock IF, de Wit R, Berry WR, Horti J, Pluzanska A, Chi $\mathrm{KN}$, et al. Docetaxel plus prednisone or mitoxantrone plus prednisone for advanced prostate cancer. N Engl J Med 2004;351:1502-12.

6. Beer TM, Armstrong AJ, Rathkopf DE, Loriot Y, Sternberg CN, Higano CS, et al. Enzalutamide in metastatic prostate cancer before chemotherapy. N Engl J Med 2014;371:424-33.

7. Scher HI, Fizazi K, Saad F, Taplin ME, Sternberg CN, Miller K, et al.; AFFIRM Investigators. Increased survival with enzalutamide in prostate cancer after chemotherapy. N Engl J Med 2012;367:1187-97.

8. Saad F, de Bono J, Shore N, Fizazi K, Loriot Y, Hirmand M, et al. Efficacy outcomes by baseline prostate-specific antigen quartile in the AFFIRM trial. Eur Urol 2015;67:223-30.

9. Loriot Y, Miller K, Sternberg CN, Fizazi K, De Bono JS, Chowdhury $S$, et al. Effect of enzalutamide on health-related quality of life, pain, and skeletal-related events in asymptomatic and minimally symptomatic, chemotherapy-naive patients with metastatic castration-resistant prostate cancer (PREVAIL): results from a randomised, phase 3 trial. Lancet Oncol 2015;16:509-21.

10. Kim CS, Theeuwes A, Kwon DD, Choi YD, Chung BH, Lee $\mathrm{HM}$, et al. The PREVAIL trial of enzalutamide in men with chemotherapy-naïve, metastatic castration-resistant prostate cancer: post hoc analysis of Korean patients. Investig Clin Urol 2016;57:174-83.

11. Merseburger AS, Hammerer P, Rozet F, Roumeguère T, Caffo $\mathrm{O}$, da Silva FC, et al. Androgen deprivation therapy in castrateresistant prostate cancer: how important is GnRH agonist backbone therapy? World J Urol 2015;33:1079-85.

12. Heidenreich A, Bastian PJ, Bellmunt J, Bolla M, Joniau S, van der Kwast T, et al.; European Association of Urology. EAU guidelines on prostate cancer. Part II: Treatment of advanced, relapsing, and castration-resistant prostate cancer. Eur Urol 2014;65:467-79.

13. Merseburger AS, Björk T, Whitehouse J, Meani D. Treatment costs for advanced prostate cancer using luteinizing hormonereleasing hormone agonists: a solid biodegradable leuprorelin implant versus other formulations. J Comp Eff Res 2015;4:44753.

14. Taylor CD, Elson P, Trump DL. Importance of continued testicular suppression in hormone-refractory prostate cancer. J Clin Oncol 1993;11:2167-72.

15. Hussain M, Wolf M, Marshall E, Crawford ED, Eisenberger M. Effects of continued androgen-deprivation therapy and other prognostic factors on response and survival in phase II chemotherapy trials for hormone-refractory prostate cancer: a Southwest Oncology Group report. J Clin Oncol 1994;12:186875. 
16. Lee JL, Eun Kim J, Ahn JH, Lee DH, Lee J, Kim CS, et al. Role of androgen deprivation treatment in patients with castrationresistant prostate cancer, receiving docetaxel-based chemotherapy. Am J Clin Oncol 2011;34:140-4.

17. Bong GW, Clarke HS Jr, Hancock WC, Keane TE. Serum testosterone recovery after cessation of long-term luteinizing hormone-releasing hormone agonist in patients with prostate cancer. Urology 2008;71:1177-80.

18. Kobayashi T, Nishizawa K, Mitsumori K. Individual variation of hormonal recovery after cessation of luteinizing hormonereleasing hormone agonist therapy in men receiving long-term medical castration therapy for prostate cancer. Scand J Urol Nephrol 2006;40:198-203.

19. Kaku H, Saika T, Tsushima T, Ebara S, Senoh T, Yamato T, et al. Time course of serum testosterone and luteinizing hormone levels after cessation of long-term luteinizing hormonereleasing hormone agonist treatment in patients with prostate cancer. Prostate 2006;66:439-44.
20. Hall MC, Fritzsch RJ, Sagalowsky AI, Ahrens A, Petty B, Roehrborn CG. Prospective determination of the hormonal response after cessation of luteinizing hormone-releasing hormone agonist treatment in patients with prostate cancer. Urology 1999;53:898-902; discussion 902-3.

21. Pinski J, Xiong S, Wang Q, Stanczyk F, Hawes D, Liu SV. Effect of luteinizing hormone on the steroidogenic pathway in prostate cancer. Prostate 2011;71:892-8.

22. O'Donnell A, Judson I, Dowsett M, Raynaud F, Dearnaley D, Mason M, et al. Hormonal impact of the 17alpha-hydroxylase/ $\mathrm{C}(17,20)$-lyase inhibitor abiraterone acetate (CB7630) in patients with prostate cancer. Br J Cancer 2004;90:2317-25.

23. Ohlmann $\mathrm{CH}$, Jäschke $\mathrm{M}$, Jaehnig P, Krege S, Gschwend J, Rexer $\mathrm{H}$, et al. Abiraterone acetate plus LHRH therapy versus abiraterone acetate while sparing LHRH therapy in patients with progressive, metastatic and chemotherapy-naïve, castration-resistant prostate cancer (SPARE): study protocol for a randomized controlled trial. Trials 2017;18:457. 